\title{
Particle-covered droplet and a particle shell under compressive electric stress
}

\author{
Khobaib Khobaib $\odot,{ }^{1}$ Tomasz Hornowski $\odot,{ }^{1}$ and Zbigniew Rozynek $\odot^{1,2, *}$ \\ ${ }^{1}$ Faculty of Physics, Adam Mickiewicz University, Uniwersytetu Poznańskiego 2, 61-614 Poznań, Poland \\ ${ }^{2}$ PoreLab, The Njord Centre, Department of Physics, University of Oslo, Blindern, N-0316 Oslo, Norway
}

(Received 15 October 2020; revised 19 March 2021; accepted 20 May 2021; published 8 June 2021)

\begin{abstract}
Understanding of the behavior of an individual droplet suspended in a liquid and subjected to a stress is important for studying and designing more complex systems, such as emulsions. Here, we present an experimental study of the behavior of a particle-covered droplet and its particle shell under compressive stress. The stress was induced by an application of a DC electric field. We studied how the particle coverage $(\varphi)$, particle size $(d)$, and the strength of an electric field $(E)$ influence the magnitude of the droplet deformation $(D)$. The experimental results indicate that adding electrically insulating particles to a droplet interface drastically changes the droplet deformation by increasing its magnitude. We also found that the magnitude of the deformation is not retraceable during the electric field sweeping, i.e., the strain-stress curves form a hysteresis loop due to the energy dissipation. The field-induced droplet deformation was accompanied by structural and morphological changes in the particle shell. We found that shells made of smaller particles were more prone to jamming and formation of arrested shells after removal of an electric stress.
\end{abstract}

DOI: 10.1103/PhysRevE.103.062605

\section{INTRODUCTION}

Droplets covered by granular or colloidal particles have recently been actively studied from the perspective of both the fundamental and the applied sciences. They are considered as suitable materials to be used in the food technology [1], drug delivery [2], cosmetics [3], biomedical applications [4], and oil industry [5]. Particle-covered droplets have characteristics that make them useful also for fabricating adaptive structures [6,7], porous materials [8], colloidal photonic crystals [9], and responsive microcapsules with homogenous $[10,11]$ or heterogeneous particle shells [12,13]. Moreover, the particlecovered droplets can be used as experimental model systems for studying different phenomena taking place on curved liquid-liquid interfaces, for example, particle assembly [14], ordering [15], mixing [16], as well as particle-layer buckling [17].

In many research areas, knowledge of the stability and mechanics of an individual particle-covered droplet is essential, e.g., for the efficient fabrication of Pickering emulsions [18], for designing emulsions with controlled stability $[19,20]$, and, in general, for the further development of the above mentioned research fields. In this context, several research groups have studied theoretically and experimentally the deformation [21-24], relaxation [25,26], dynamics [27], and mechanical properties of particle-laden droplets $[28,29]$. Experimen-

\footnotetext{
*Corresponding author: zbiroz@amu.edu.pl
}

Published by the American Physical Society under the terms of the Creative Commons Attribution 4.0 International license. Further distribution of this work must maintain attribution to the author(s) and the published article's title, journal citation, and DOI. tal methodologies involved in research on particle-covered droplets include compressive strain [25,30], atomic force microscopy [31], as well as ultrasonic and magnetic methods $[32,33]$. Another approach for studying the properties of particle-laden droplets is the application of an electric field ( $E$ field).

$E$ fields have demonstrated to be an adaptable method for studying particle-droplet stability [20], electrorotation $[16,22]$, as well as steady-state $[34,35]$ and transient deformations [36-38]. In this paper, we use an $E$ field to study the behavior of a particle-covered oil droplet and its particle shell under induced electric stress. In the oil-oil systems, an $E$ field can be used to generate the electric stresses that either compress or stretch a particle-covered droplet depending on physical parameters of the fluids and particles (e.g., electrical conductivity and dielectric properties), and the parameters of an applied $E$ field (frequency and strength). For example, a particle-covered droplet subjected to a DC $E$ field can compressively deform, whereas an application of an AC $E$ field to the same droplet may stretch it [6]. Here, we wished to investigate a particle-covered droplet under compressive stress. Therefore, we chose to use a uniform DC $E$ field and work with a three-phase system comprising an electrically weakly conductive silicone oil droplet covered by electrically insulating microparticles and suspended in slightly more electrically conductive castor oil. In such a system, the $E$ field causes free ions with opposite charges (impurities in the oils) to accumulate at the two hemispheres of the particle-covered droplet. Electric stress is induced at the hemispheres when the $E$ field acts on these charges, and it compresses the droplet that eventually acquires an oblate geometry, i.e., the longest droplet axis is perpendicular to the $E$ field.

Many experimental and theoretical studies have been conducted on this type of deformation. Initially, the majority 
of the studies concerned particle-free droplets. The theory on the droplet deformation and relaxation (after reducing $E$-field strength) was established a long time ago [39] and has since been further developed [37,40-43]. Thus, the influence of liquids' electrical properties and parameters of the $E$ field on the mechanics of pure droplets is now well described. Much less is known about the deformation of particle-covered droplets, although, lately the knowledge gap has been narrowing by the works of several research groups [6,24,34-36,44]. However, in all these research contributions the particle-covered droplets were studied in a narrow range of $E$-field strengths. This is because the particle-covered droplets suspended roughly in the middle of the sample cell and unattached to any surface could freely translate and rotate in the presence of an $E$ field. Thus, at moderate strengths of $E$ fields (above $\sim 150 \mathrm{~V} \mathrm{~mm}^{-1}$ ) a particle-covered droplet begins electrorotating, which prevents researching on the droplet deformation at higher $E$-field strengths. In the research presented here, we solve this problem by docking a droplet into an O-ring washer attached to one of the walls of the sample cell. In this way the droplet is held in place preventing: (i) its motion in the sample cell due to the presence of convective flows of liquid in the cell and (ii) its electrorotation as well as easing the experimental observations. Therefore, we can study the behavior of a droplet and its particle shell subjected to much greater $E$-field strengths $\left(\sim 300 \mathrm{~V} \mathrm{~mm}^{-1}\right)$.

There is also little knowledge about the influence of particle coverage on the droplet deformation. In this paper, we investigate thoroughly this correlation. Another subject of consideration is the relaxation of the particle-covered droplets. Numerous research articles deal with the relaxation kinetics $[25,35,44,45]$. Lately, several works have been published on the behavior of the particle shell after removal of the external force. The research concerned particle jamming (leading to formation of stable nonspherical droplets), particle shell buckling, and structural changes [46-49]. In our previous work on opening and closing of particle shells on droplets, we found that the magnitude of the droplet deformation was nonretractable and exhibited hysteresis, and the particle shell underwent structural and morphological changes [46]. Rane et al. [30] reported hysteresis in deformation of liquid marbles during the mechanical compression and decompression. They attributed the presence of the hysteresis to the interlocking and rearrangement of particles at the droplet interface. Monteux et al. [50] and Xu et al. [51] also demonstrated the hysteresis effect by volume compression and expansion of the droplet, and they attributed the slow rearrangement due to the particles aggregation at the droplet interface.

Different mechanisms of droplet compression and decompression were used in the four above mentioned works, and the force on a particle-covered droplet was exerted in a different way. This has certain consequences. For example, during the mechanical compression of a droplet by two flat slabs, the particles in the shell become loosely packed. Whereas, in the method presented here and in our previous work [46], the particles are kept tight during the application of the $E$ field, which may result in buckling of the shell. Due to the limited understanding of our previous result, we decided to conduct further research.
Thus, in this paper, our objectives are to show: (i) how the particle coverage, particle size, and the strength of the $E$ field influence the magnitude of steady-state deformation of a droplet with a particle shell; and (ii) how the particle size and the strength of the $E$ field affect the recovery of a particle shell and the arrangements of the surface particles.

\section{EXPERIMENTAL SETUP AND MATERIALS}

The experimental setup consisted of an optical acrylic cuvette $\left(10 \times 10 \times 30 \mathrm{~mm}^{3}\right)$ used as a sample cell with two copper plates that constituted electrodes, a signal generator (SDG1025, SIGLENT Technologies), a high voltage amplifier for generating a direct current electric signal (10HVA24-BP1, HVP High Voltage Products $\mathrm{GmbH}$ ), and a digital microscope (AM7315MZT, Dino-Lite) for viewing perpendicular to the direction of the applied $E$ field. A schematic illustrating the experimental setup is shown in Figs. 1(a) and 1(b). A plastic washer (outer diameter $4.0 \mathrm{~mm}$, inner diameter $2.3 \mathrm{~mm}$, and thickness $0.4 \mathrm{~mm}$ ) was glued to one of the copper electrodes (at its center). The washer was used to hold a droplet in place, i.e., prevent: (i) its motion in the sample cell in the presence of convective flows of liquid and (ii) its rotation [22]. The presence of the washer influenced only slightly (by less than $15 \%$ ) the magnitude of the droplet deformation (see Fig. S1 of the Supplemental Material [52]). We, therefore, do not take it into account in the data analysis and discussion. In the experiments with results presented in Figs. 8 and 9, we used a cell made of glass with two glass walls coated by an electrically conductive indium tin oxide (ITO) layer.

Polyethylene particles (WPMS-0.98, size $\sim 3 \mu \mathrm{m}$, density $\sim 0.98 \mathrm{~g} \mathrm{~cm}^{-3}$; GPMS-0.98, size $\sim 18 \mu \mathrm{m}$, density $\sim 0.98 \mathrm{~g} \mathrm{~cm}^{-3}$; $\quad$ BLPPMS-1.00, size $\sim 30 \mu \mathrm{m}$, density $\sim 1.00 \mathrm{~g} \mathrm{~cm}^{-3}$; REDPMS-0.98, size $\sim 50 \mu \mathrm{m}$, density $\sim 0.98 \mathrm{~g} \mathrm{~cm}^{-3} ; \quad$ GPMS- $0.98, \quad$ size $\sim 100 \mu \mathrm{m}$, density $\sim 0.98 \mathrm{~g} \mathrm{~cm}^{-3}$; electrical conductivity $\sim 10^{-15} \mathrm{~S} \mathrm{~m}^{-1}$, purchased from Cospheric LLC) and clay mineral particles (Li fluorohectorite, size $\sim 5 \mu \mathrm{m}$; density $\sim 2.8 \mathrm{~g} \mathrm{~cm}^{-3}$, Corning, Inc., USA) were used to make a monolayer shell on a silicone oil (VWR Chemicals, Rhodorsil® 6678.1000, density $0.96 \mathrm{~g} \mathrm{~cm}^{-3}$, electrical conductivity $\sim 10 \mathrm{pS} \mathrm{m}{ }^{-1}$, relative permittivity $\sim 2.6-2.9$ at $25^{\circ} \mathrm{C}$, and viscosity $50 \mathrm{mPa}$ s) droplet formed in castor oil (Sigma-Aldrich 83912 , density $0.961 \mathrm{~g} \mathrm{~cm}^{-3}$ at $25^{\circ} \mathrm{C}$, electrical conductivity $\sim 60 \mathrm{pS} \mathrm{m}^{-1}$, relative permittivity $\sim 4.6-4.8$ at $25^{\circ} \mathrm{C}$, and viscosity 750 $\mathrm{mPa}$ ). The interfacial tension between the two immiscible oils was around $4.5 \mathrm{mN} \mathrm{m}^{-1}$.

\section{FORMATION OF PARTICLE-COVERED DROPLETS AND EXPERIMENTAL PROCEDURES}

We used an $E$-field approach (see Ref. [7]) to form a monolayer particle shell on the surface of a silicone oil droplet. Initially, silicone oil dispersion of polyethylene (PE) particles was prepared using a specific particle concentration required to form a particle-covered droplet of a certain size and a desired particle coverage. The dispersion was ultrasonicated for $5 \mathrm{~min}$ to avoid particle aggregating, and, subsequently, a dispersion droplet was formed (using a regular mechanical pipette) in a cuvette filled with castor oil. Next, an $E$ field 

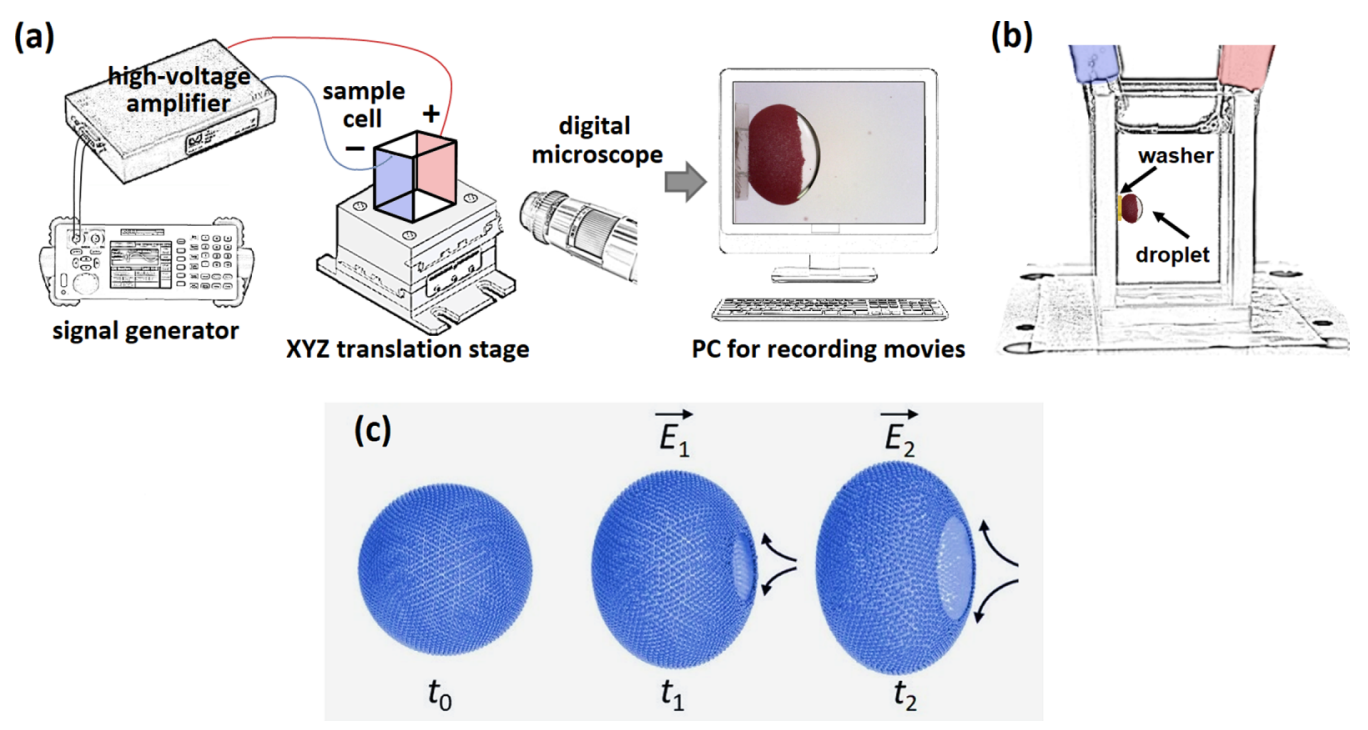

FIG. 1. (a) and (b) Schematics illustrating the experimental setup, which consisted of a digital microscope for viewing perpendicular to the direction of the applied $E$ field, a sample cell placed on a mechanical XYZ translational stage, a signal generator, and a voltage amplifier for generating a high-voltage electric signal, which is provided to the electrodes inserted inside the sample cell. (c) Schematics of particle shell opening: A droplet with the surface particles is viewed at a $30^{\circ}$ angle with respect to the $E$-field direction, which is horizontal. Application of an electric field $\left(E_{1}\right)$ to the initially spherical droplet $\left(t_{0}\right)$ densely covered with particles results in its deformation due to the electric stress. The induced EHD flows convect particles away from the droplet's electric pole thereby forming an opening in the particle layer. After application of a stronger electric field $\left(E_{2}\right)$, the droplet deforms more, allowing the opening to grow in size, and the EHD flows to strengthen (indicated by the longer curved arrows).

$\left(\sim 200 \mathrm{~V} \mathrm{~mm}^{-1}\right)$ was applied so that the particles in the bulk liquid of the dispersion droplet were guided toward the droplet's interface by electrostatic force. As the particles reached the surface of the droplet, they were carried toward the electric equator of that droplet by the $E$-field induced liquid flows. It took several minutes for all particles to get onto the interface and eventually form a packed particle monolayer. The particles were irreversibly trapped at the droplet interface by capillary forces as the thermal energy is a few orders of magnitude smaller than that stemming from the capillary interaction for microparticles [48]. The droplet was then docked in an O-ring washer to prevent its motion in the sample cell and ease the experimental observations. To avoid particles inside the ring, we first docked a pure silicone oil droplet and then brought the particle-covered droplet and let it electrocoalesce. To adjust the droplet volume and the particle coverage, some of the silicone oil was extracted from the droplet using a micropipette. This spherical particle-covered droplet was then studied in an $E$ field, which was in the horizontal direction.

When a particle-free droplet or a droplet covered with PE particles is subjected to a direct current $E$ field, free charges (ionic impurities in oils) accumulate at the droplet's interface. This results in the generation of the electric stress that deforms the droplet, thereby increasing the droplet's surface area and decreasing the particle coverage. The steady-state deformation of the droplet due to the electric stress is given by the Melcher-Taylor model [53],

$$
D=\frac{9 r_{0} \varepsilon_{0} \varepsilon_{\mathrm{ex}} E_{0}^{2}}{16 \gamma S(2+R)^{2}}\left[S\left(R^{2}+1\right)-2+3(R S-1) \frac{2 \lambda+3}{5 \lambda+5}\right],
$$

where $R=\frac{\sigma_{\mathrm{in}}}{\sigma_{\mathrm{ex}}}, S=\frac{\epsilon_{\mathrm{ex}}}{\epsilon_{\mathrm{in}}}, \lambda=\frac{\mu_{\mathrm{ex}}}{\mu_{\mathrm{in}}}$, and $R, S$, and $\lambda$ are the conductivity, dielectric constant, and viscosity ratios, $\varepsilon_{\mathrm{ex}}$ is the dielectric constant of the surrounding fluid, $r_{0}$ is the radius of the droplet, and $\gamma$ is the interfacial surface tension between the droplet and the exterior fluid. The subscript "ex" represents the exterior fluid (castor oil), whereas the subscript "in" represents the interior fluid which is the droplet (silicone oil). In the system studied here, the electric stress compresses the droplet, therefore, the magnitude of the deformation has a negative sign if the deformation is described as $D=\left(d_{\|}-d_{\perp}\right) /\left(d_{\|}+d_{\perp}\right)$, where $d_{\|}$and $d_{\perp}$ are the droplet's axes parallel and perpendicular to the $E$-field direction, respectively. However, in all our plots we use the absolute value of $D$. Application of the $E$ field also results in the induction of the electrohydrodynamic (EHD) flows. In the case of the particle-covered droplets, these flows convect particles away from the droplet's electric pole, forming a particle-free area there. The convected particles pack densely within the particle shell. When the $E$-field strength is increased, the droplet deforms more, allowing the particle-free area to grow in size and the EHD flows to strengthen as presented in Fig. 1(c).

In our experiments, we varied the $E$-field strength and the particle coverage as well as the droplet size and studied changes in the magnitude of the droplet's deformation by estimating the major and minor lengths of the droplet using GRAPHS software. We limited the $E$-field strength to $285 \mathrm{~V} \mathrm{~mm}^{-1}$ because at stronger $E$ fields (above $\sim 300 \mathrm{~V} \mathrm{~mm}^{-1}$ ) particles detached irreversibly from the droplet's surface. The particles coverage was also estimated through image analysis of the droplets. We define here the particle coverage of the droplets as $\varphi=S / A$, where $S$ is the 
surface area of the particle film and $A$ is the surface area of the droplet with excluded part of the droplet in the washer. Thus, the value of the particle coverage as defined here is in the range from 0 to around 1.0. $\varphi=0$ defines the silicone oil droplet without particles, and $\varphi=1$ defines the silicone oil droplet fully covered by the PE particles.

\section{PARTICLE IMAGE VELOCIMETRY EXPERIMENTS}

The flowlines around droplets were traced using particle image velocimetry (PIV). Fluorescent PE particles (UVPMSBY2-1.00, size of $\sim 35 \mu \mathrm{m}$, Cospheric LLC) were dispersed in castor oil ( $0.3 \%$ by weight) and poured in the sample cell. A $500-\mu \mathrm{m}$ thin sheet of particles was selected by focusing a 532-nm laser light (COM-09906-5 mW, SparkFun Electronics) from above the sample cell through a laser line generator lens (Powell lens), similar as in the experiment presented in Ref. [6]. During the experiments, the laser sheet was always aligned in the middle of the drop, oriented along the $E$-field direction, and perpendicular to the view direction of the microscope (see Fig. S2 in the Supplemental Material [52]). Movies (5 fps) were recorded for each experiment with a $1920 \times 1080$ -pixel resolution ( 1 pixel $\sim 6.2 \mu \mathrm{m}$ ). Twenty-five sequential frames were then compared and analyzed using PIVLAB (v.1.41, MATLAB toolbox application), yielding flow velocities for each interrogation area (the frames were split into a number of interrogation areas of size $64 \times 64$ pixels, which were then individually cross-correlated with the previous frame to obtain displacement vectors). MATLAB (v.R2017b, MathWorks) was then used to plot the flow velocities.

\section{RESULTS}

General concept of the experiments: In all the experiments we used a tabletop experimental setup, which consisted of a sample cell with two electrodes, a source of a high voltage DC signal, and a digital microscope for viewing either parallel or perpendicular to the direction of the applied $E$ field. A schematic illustrating part of the experimental setup is shown in Figs. 1(a) and 1(b). Application of a DC $E$ field results in a compressive electric stress that acts on particle-covered droplet deforming it. When the droplet deforms, its surface area increases leading to unjamming of particles in a shell. This, in turn, enables the induction of the EHD flows. In our system, these flows convect particles away from the droplet's electric pole, forming a particle-free area there. The convected particles pack densely within the particle shell. When the $E$ field strength is increased, the droplet deforms more, allowing the particle-free area to grow in size and the EHD flows to strengthen as presented in Fig. 1(c).

\section{A. Influence of particle coverage on the droplet deformation and emergence of its hysteresis}

We began our research with studying the influence of particle coverage on the magnitude of steady-state droplet deformation at different strengths of an $E$ field. We prepared seven silicone oil droplets $(\sim 4 \mathrm{~mm})$ covered by the PE particles $(\sim 50 \mu \mathrm{m})$, each droplet with different particle coverage. We also used a particle-free droplet as a reference. Initially, in the absence of the $E$ field, the droplets were spherical. Application of the $E$ field induced electric stress on the droplets and resulted in their deformation, i.e., all droplets developed an oblate shape. The strength of the $E$ field was increased stepwise from $0 \mathrm{~V} \mathrm{~mm}^{-1}$ to $285 \mathrm{~V} \mathrm{~mm}^{-1}$ and then decreased in the same manner to $0 \mathrm{~V} \mathrm{~mm}^{-1}$. At each step, the $E$-field strength was increased by $15 \mathrm{~V} \mathrm{~mm}^{-1}$ and maintained until a steady state (droplet's deformation and particle arrangement) was observed. The difference in the droplet deformation can be easily observed qualitatively, especially at strong $E$ fields, for example, at $285 \mathrm{~V} \mathrm{~mm}^{-1}$ as presented in Fig. 2(a). The droplets with higher particle coverage deform more. In Figs. 2(b)-2(d), we present the quantitative data from the experiments, which reveal the nonobvious behavior of particle-covered droplets.

In Fig. 2(b), the magnitude of droplet deformation is plotted against the square of the $E$-field strength. As expected, the magnitude of the deformation of the pure silicone oil droplet scales as $E^{2}$ at weak $E$ fields (up to around $15 \mathrm{kV}^{2} \mathrm{~mm}^{-2}$ )) and follows Taylor's theory [39,54]. At stronger $E$ fields, the curve becomes nonlinear and bends downward. The reason for this nonlinearity is the surface-charge convection (not included in Taylor's theory) due to the EHD liquid flows making it more difficult to deform the droplet into more oblate shape as explained and experimentally presented in Refs. [40-42,55]. The curve for the pure silicone oil droplet is entirely retraceable when the $E$ field is decreased back to zero, i.e., the data points $(\diamond)$ overlap when sweeping the $E$ field up and down.

A different situation is observed for the droplet covered with particles. The magnitude of the deformation is not retraceable (i.e., there are different values of the deformation depending on the direction of change of the $E$-field strength) and the curves form a hysteresis loop (will come back to that later). In addition, the magnitude of droplet deformation is sensitive to particle coverage. In general, the deformation is greater with the increased particle coverage. In Fig. 2(c), we plot the deformations measured at the strongest $E$ field used in the experiment $\left(285 \mathrm{~V} \mathrm{~mm}^{-1}\right)$ versus the particle coverage $(\varphi)$. It can be seen that the magnitude of the deformation does not change much for the droplets with low particle coverage but increases sharply at higher particle coverages.

There are possibly two reasons for this: (i) As more of the droplet's surface is covered by particles, the charge convection may be depleted due to the reduced strength of EHD flows leading to accumulation of a larger amount of free charges on the droplet's surface. This, in turn, results in stronger electrical stress acting on the droplet. In addition, (ii) the effective electrical conductivity of the droplet interface may decrease (because the PE particles are several orders of magnitude less conductive than silicone oil [44]), leading to the greater electrical contrast between the particle-covered silicone oil droplet and castor oil and, thus, enhancing the compressive electric stress acting on the droplet. We conducted experiments and performed calculations to confront the above statements.

In Fig. 3, we present the results of PIV experiments performed on a silicone oil droplet with PE particle particles $(\sim 50 \mu \mathrm{m})$ at different particle coverages subjected to a DC $E$ field of $170 \mathrm{~V} \mathrm{~mm}^{-1}$. The results from the PIV experiments confirm that the induced EHD flows at droplet interfaces are 

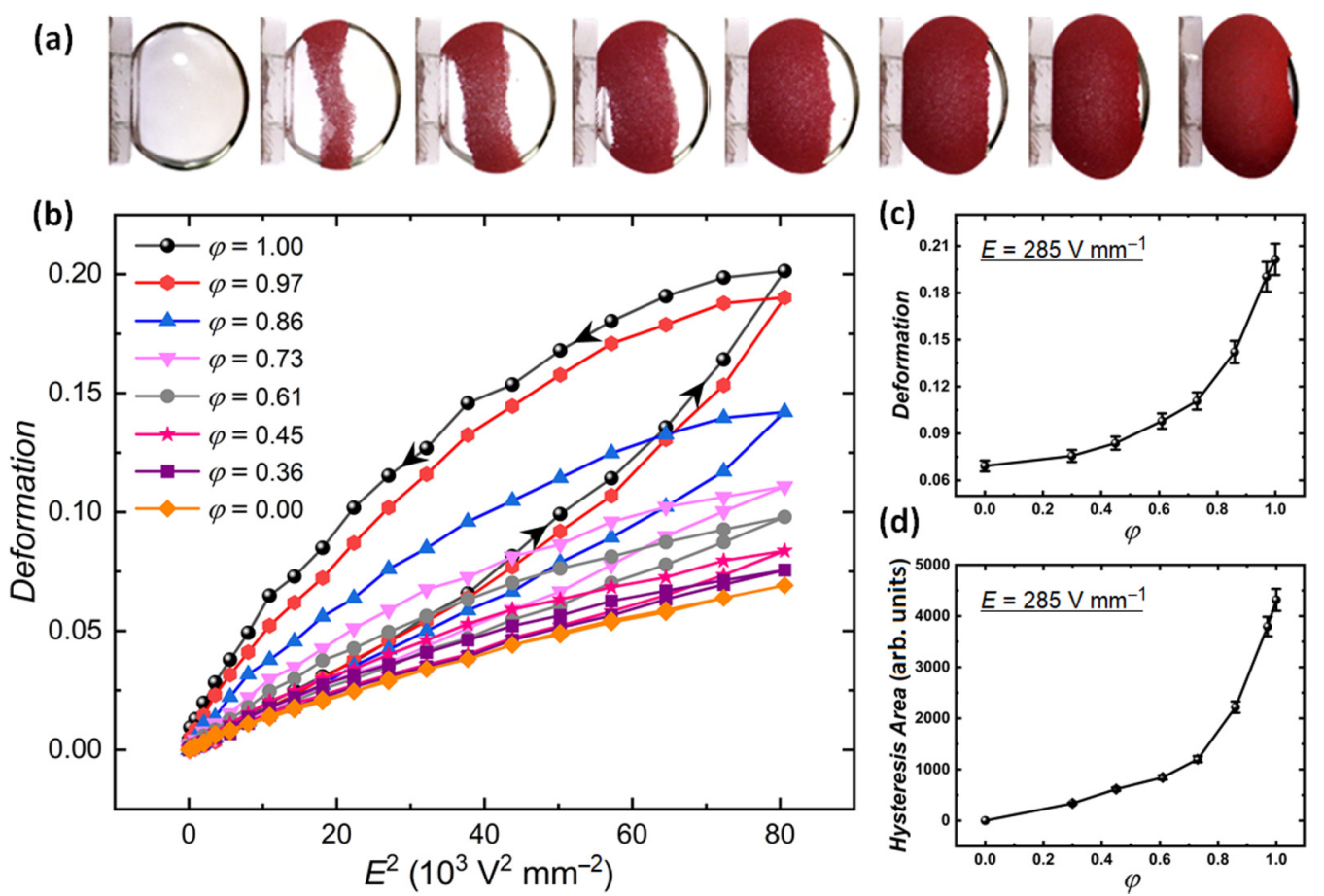

FIG. 2. (a) Silicone oil droplets with different particle coverages $(\varphi)$ subjected to $E$-field strength of $285 \mathrm{~V} \mathrm{~mm}^{-1}$. The diameter of each droplet is $\sim 4 \mathrm{~mm}$, and the size of the PE particles is $\sim 50 \mu \mathrm{m}$. (b) Steady-state deformation of droplets plotted as a function of the square of the applied DC $E$ field. The $E$ field was increased stepwise from 0 to $285 \mathrm{~V} \mathrm{~mm}^{-1}$ and then decreased stepwise to $0 \mathrm{~V} \mathrm{~mm}^{-1}$. The black arrows represent the $E$-field sweep direction. (c) The magnitude of the droplet deformation and (d) the hysteresis area of the droplet deformation at $E=285 \mathrm{~V} \mathrm{~mm}^{-1}$ plotted as a function of particle coverage.

indeed greatly reduced by adding surface particles. When the particle coverage was increased from 0.25 to around 0.9 (droplet nearly fully covered by particles), the maximum EHD flow velocity decreased from around 130 to nearly $0 \mu \mathrm{m} \mathrm{s}^{-1}$, verifying that the straining flows surrounding a silicone oil droplet in castor oil are suppressed when the droplet is fully
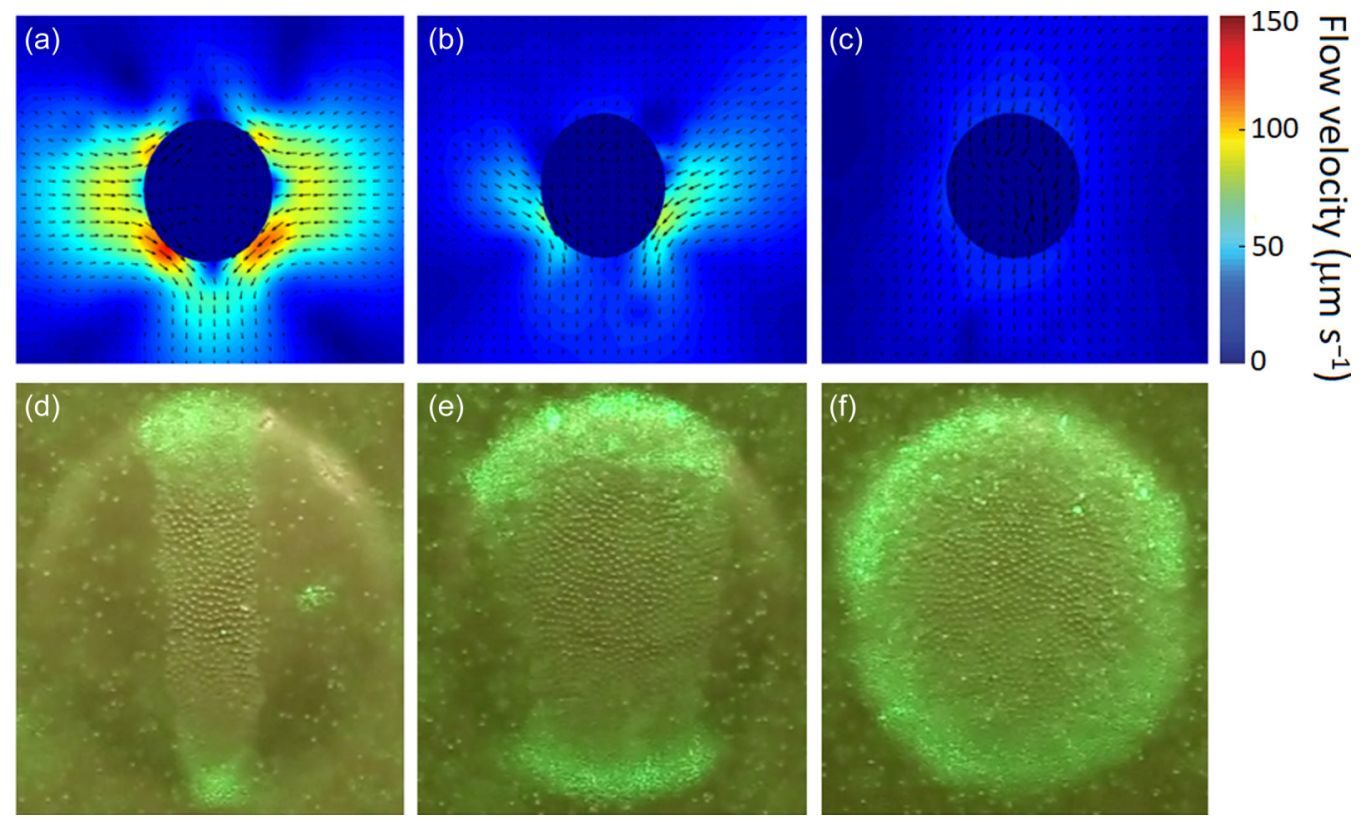

FIG. 3. (a)-(c) PIV images and (d)-(f) corresponding pictures of silicone oil droplets (diameter $\sim 2$ mm) covered with PE particles (size $\sim 50 \mu \mathrm{m}$ ). The droplets were formed in a dispersion of tracer particles $(\sim 35 \mu \mathrm{m})$ in castor oil and subjected to an $E$-field strength of $170 \mathrm{~V} \mathrm{~mm}^{-1}$ (in the horizontal direction) yielding steady-state droplet deformations. The asymmetry of the flow fields in (a) and (b) was caused by a slow droplet sedimentation causing slightly nonuniform particle distribution (more particles on the top of a droplet). The particle coverage was (a) and (d) $\sim 0.25$, (b) and (e) $\sim 0.55$, and (c) and (f) $\sim 0.9$. 

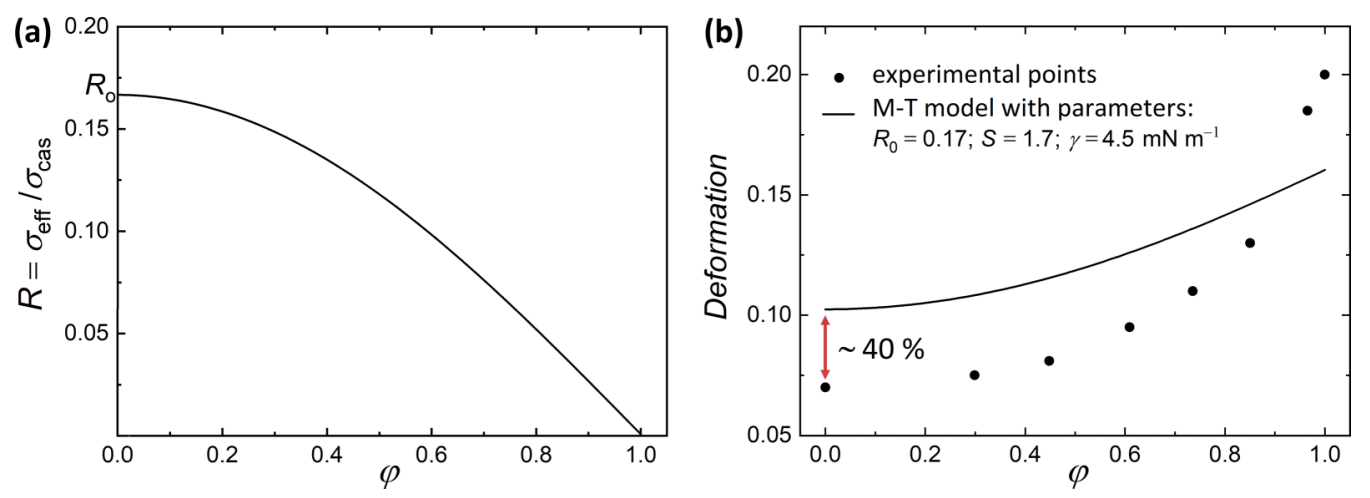

FIG. 4. (a) The electrical conductivity ratio $(R)$ plotted as function of the particle coverage $(\varphi)$. (b) The magnitude of the droplet deformation $(D)$ plotted as a function of droplets' particle coverage. The solid line is obtained by using the Melcher-Taylor equation in which the trigonometric dependency for the effective electrical conductivity [see panel (a)] is included.

covered by the particles. This leads to the buildup of a greater number of charges at the droplet's interface, which, in turn, deforms the droplet more.

We will now estimate the change in the effective conductivity of the particle covered droplet and examine its effect on the magnitude of droplet's deformation. We recall that the EHD flows convect the particles towards the electric equator. Thus, by adding particles to the droplet's interface, the particle shell widens towards the droplet's electric pole. The contribution of the particles' conductivity to the effective electric conductivity of the droplet's interface is practically zero for those particles residing at the droplet's electric equator. We know that the surface charge density distribution on a droplet scales as $\cos (\theta)$ [55], where $\theta$ is the angle measured from the zenith direction. Therefore, the particles nearest to the droplet's electric pole should contribute the most to the change in the effective conductivity. We, thus, attempt to represent the effective conductivity by the cosine function,

$$
\sigma_{\text {eff }}(\varphi)=\sigma_{\text {sil }} \cos \left(C_{1} \frac{\pi}{2} \varphi\right),
$$

where the prefactor $C_{1}=0.9999$ is introduced to obtain the finite value of the conductivity when $\varphi=1$, which is here the conductivity of PE particles. For simplicity, we assumed that $\varphi$ scales linearly with $\theta$, and $\theta$ is in the range of $0-90^{\circ}$ for $\varphi$ in the range of $0-1$. The calculated values of the conductivity ratio $(R)$, using Eq. (2), are plotted in Fig. 4(a). Taking into account this trigonometric dependency in the Melcher-Taylor equation [Eq. (1)], we calculated values of $D(\varphi)$ and plotted them in Fig. 4(b) (see the solid black curve).

The theoretical results capture the upward bending trend with the particle coverage. Although, the magnitude of deformation is overestimated for $\varphi$ values in the low range. This is what we expected: The Melcher-Taylor model does not take into account straining flows present at low particle coverage. The straining EHD flows affect the deformation by reducing its magnitude. As presented in Ref. [40] (see Fig. 7), the magnitude of droplet's deformation can be reduced by around $30 \%$ compared to that estimated from the Melcher-Taylor model. In our case the value of the droplet's deformation is smaller by around $40 \%$ compared to the calculated valued. The reason for this could be the experimental procedure in which the droplet is docked into the washer. As mentioned before, docking the droplet into the washer results in reduced magnitude of deformation by up to $15 \%$. As the particle coverage increases, the difference between the experimental and the theoretical points decreases, and at high $\varphi$ values, the magnitude of deformation is underestimated. This is because the Melcher-Taylor theory does not represent well such large deformations. In addition, the presence of surface particles may influence the surface tension. Ouriemi and Vlahovska [22] showed that the surface tension can be significantly reduced by adding particles to the droplet's interface, which resulted in the increased magnitude of the deformation. Overall, the tendency illustrated in Fig. 4(b) confirms the above stated hypothesis that the decreased effective electrical conductivity of the droplet interface enhances the compressive electric stress acting on the droplet. However, nontrivial numerical calculations would be needed to better represent the experimental data.

We also observed the emergence of the hysteresis loops as the particle coverage was increased [see Fig. 2(d)]. The hysteresis loop area does not change much for the droplets with low particle coverage. However, above coverage of around 0.65 the increase is sharp. The hysteresis loop is an indication of the energy dissipation. In the studied system, the energy can be dissipated through heat and viscous drag. The decoupling of these contributions and the estimation of the amounts of energy loss from the heat dissipation and the viscous drag is nontrivial and is beyond the scope of this paper. Nevertheless, we note that during the droplet compression and recovery, the particles relocate separately or through particle gliding and rotate dissipating the energy. In addition, (as it will be presented in the section Jamming of a particle shell) shells composed of smaller particles ( $5 \mu \mathrm{m}$ or smaller) undergo fracturing, which indicates that they form cohesive films, which may affect droplet's deformation and relaxation. We, therefore, decided to study this more closely by investigating the role of particle size on droplet deformation and relaxation.

\section{B. Influence of particle size on droplet deformation and recovery of a particle shell}

We performed an experiment with five silicone oil droplets ( $\sim 4 \mathrm{~mm}$ ), each covered with different sizes of PE particles (from 3 to $100 \mu \mathrm{m}$ ). In the experiment, the $E$ field was swept $0 \leftrightarrow 270 \mathrm{~V} \mathrm{~mm}^{-1}$ in the same manner as in the previous 


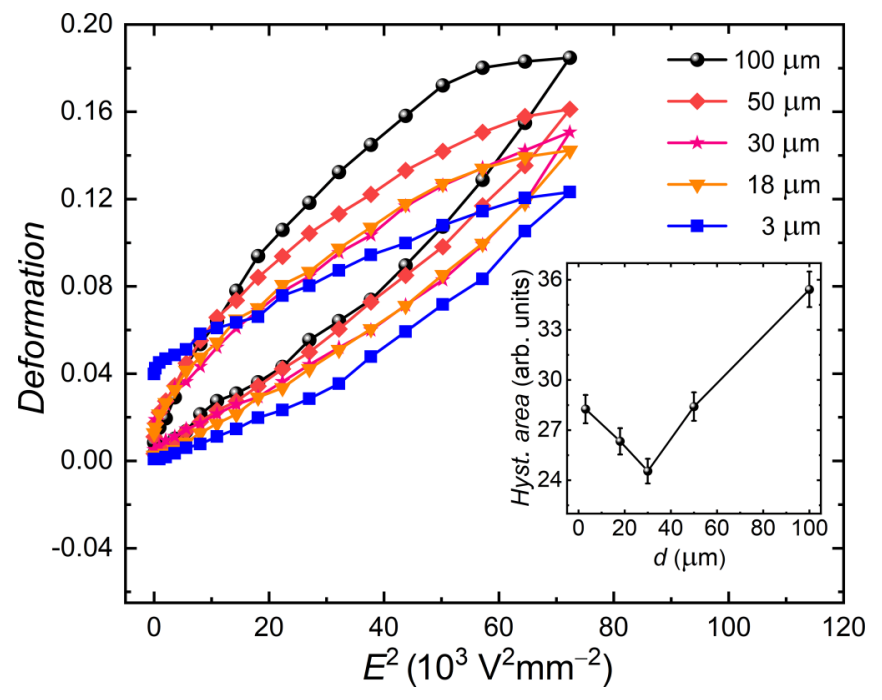

FIG. 5. Effect of the particle size on the hysteresis area of droplet deformation: Five silicone oil droplets $(\sim 4 \mathrm{~mm})$, each covered with different sizes of PE particles (from 3 to $100 \mu \mathrm{m}$ ) subjected to $E$ fields. The magnitude of steady-state deformation of the droplet plotted against the square of the $E$ field that was increased stepwise from 0 to $270 \mathrm{~V} \mathrm{~mm}^{-1}$ and then decreased stepwise to $0 \mathrm{~V} \mathrm{~mm}^{-1}$. The inset plot shows the estimated hysteresis area plotted against the particle size. The data reveal the threshold of particle size $(30 \mu \mathrm{m})$, below which the hysteresis area grows due to the presence of residual deformation of a particle-covered droplet (when the $E$ field is turned off).

experiment. The results presented in Fig. 5 show that the magnitude of droplet deformation is consistently lower for the droplet covered with the smaller particles at all $E$-field strengths.

This result was surprising for us-we expected the opposite trend, i.e., the magnitude of droplet deformation to be consistently lower for droplets covered with the bigger particles. We initially assumed that the tangential component of the electric stress (everywhere on the droplet's interface, except the droplet's electric pole and equator, the electric force acting on accumulated free charges has two components: normal and tangential) should be balanced by the bending stiffness of the elastic particle shell that would deform out of plane. In such a case, shells made of particles with larger diameter (d) should withstand greater electric stress as the bending stiffness $(B)$ of a particle monolayer formed on liquid-liquid or liquid-air interface scales as $B \sim d^{2}$ [48,56]. However, after taking a closer look on what was happening with particles at the droplet's electric pole (we viewed the droplet subjected to $E$ fields with a direction along the $E$ field), we understood that particle interlocking and/or rearranging had to play an important role. Particle shells made of small particles are typically more cohesive comparing to a shell formed of larger particles. Thus, it should be more difficult to rearrange particles during the compression and, hence, more difficult to deform the droplet covered by smaller particles.

The increased magnitude of the deformation for larger particles may also have an electric origin. Therefore, we decided to perform calculations and estimate the contribution of the particle size to the change in the electrical conductivity of the droplet's interface and, thus, to the magnitude of droplet deformation. The heterogeneous electrical characteristics of the particle-covered droplet, such as particle shell's electrical conductivity and electrical conductivity of the droplet can be replaced by the equivalent homogeneous electrical conductivity $\sigma_{\text {eq }}$ using the expression $[57,58]$,

$$
\begin{aligned}
\sigma_{\mathrm{eq}} & =\sigma_{\mathrm{PE}} \frac{2(1-\beta) \sigma_{\mathrm{PE}}+(1+2 \beta) \sigma_{\mathrm{sil}}}{(2+\beta) \sigma_{\mathrm{PE}}+(1-\beta) \sigma_{\mathrm{sil}}}, \\
\beta & =\left(1-\frac{d}{2 a}\right)^{3},
\end{aligned}
$$

where, $d$ is the polyethylene particle diameter and $a$ is the radius of the droplet.

According to Eq. (3), the conductivity ratio $\left(R=\sigma_{\text {eq }} / \sigma_{\text {cas }}\right)$ decreases with the particle diameter as illustrated in Fig. 6(a). In Fig. 6(b), we plot the droplet deformation (subjected to the $E$-field strength of $270 \mathrm{~V} \mathrm{~mm}^{-1}$ ) as a function of particle radius plotted using Melcher-Taylor's equation [Eq. (1)] with the conductivity ratio. Although the deformation increases with the increase in particle radius, the calculated difference in the magnitude of deformations due to the particle size is much smaller than that observed experimentally (Fig. 5). Thus, the mechanical properties of the particle shell seem to dominate over the electric contribution or a different model has to be used than the membrane model [Eq. (3)].

As can be observed in Fig. 5, the strain-stress curve is not a complete hysteresis loop for the droplet covered with the smallest particles ( 3 and $18 \mu \mathrm{m}$ ). In the inset of Fig. 5, we plot the hysteresis area against the particle size. From that plot we learn that there is a particle size threshold below which, the hysteresis area grows due to the presence of residual deformation of a particle-covered droplet (when the $E$ field is turned off). For better comprehension of the origin of this behavior, we performed experiments in which we studied the effect of different $E$-field strengths and particle sizes on a droplet's deformation. Four droplets, each covered with particles of different average size ranging from 3 to $100 \mu \mathrm{m}$, were subjected to six $E$-field cycles, each cycle with a different maximum field strength.

In Figs. 7(a) and 7(b), we plotted the magnitude of droplet deformation against the square of the applied $E$ field for two (out of four) droplets with shells composed of 100- and 3- $\mu \mathrm{m}$ $\mathrm{PE}$ particles, respectively. As expected, the hysteresis area increased with the increase of the $E$-field strength-the more work performed on the particle-covered droplet, the more energy is dissipated. However, when sweeping the $E$ field down to zero the curves did not come back to their original points. This is particularly apparent for the droplet covered with the smallest particles [see Fig. 7(b)]. It turns out that the magnitude of the residual deformation $\left(\Delta D=d_{\mathrm{r}}-d_{\mathrm{i}}\right.$, where $d_{\mathrm{i}}$ and $d_{\mathrm{r}}$ are the shapes of the initial droplet before application of the $E$ field and the relaxed droplet after the sweeping cycle, respectively) depends on both the maximum $E$-field strength and the particle size. In Fig. 7(c), we plotted the residual deformation $(\Delta D)$ against the maximum strength of the $E$ field applied during the sweeping. The results demonstrate that the magnitude of $\Delta D$ generally increases continuously with the increase in the maximum strength of the $E$ field. The data for the three droplets for which we see the residual deformation is 

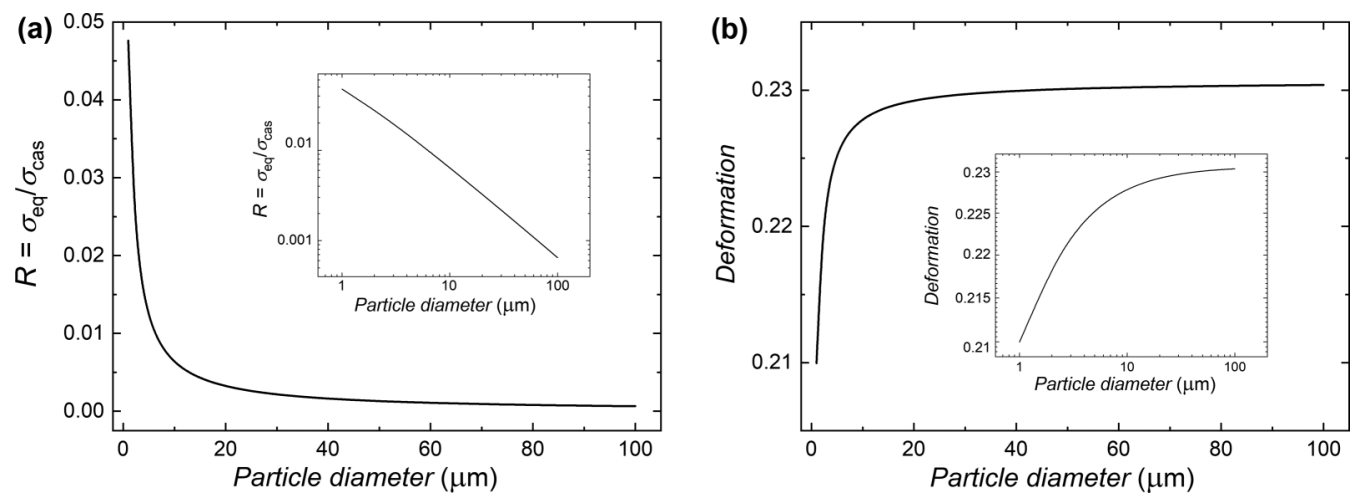

FIG. 6. (a) The electrical conductivity ratio $(R)$ plotted as function of the particle diameter $(d)$. The equivalent conductivity of the particles shell changes with the thickness of the film as expressed by Eq. (3). (b) The magnitude of the droplet deformation $(D)$ is plotted as a function of the particle diameter $(d)$. The inset figures in both panels are the log-log plots.

also presented in a log-log plot of $\Delta D$ versus $D$ [see Fig. 7(d)]. The plot reveals that $\Delta D$ is ultimately proportional to $D$ (the slope of 1 is included for a reference) within the used strengths of the $E$ field. In addition, $\Delta D$ increases with the decrease in the particle size. This may indicate that the particles within the shell undergo structural changes and jam before the droplet fully relaxes. When lowering the $E$-field strength, the particles move on the curved surface back towards the droplet's electric pole. To be able to accommodate the particles and pack them densely, they need to move and rearrange without any restrictions. Otherwise, the particles jam and form an arrested shell, preventing the droplet from returning to its spherical shape. We decided to further study the jamming of particles by viewing the droplet along the $E$-field direction.

\section{Jamming of a particle shell}

In Fig. 8, we present the results of an experiment on three droplets covered with PE particles of different sizes (diameter 3,18 , and $100 \mu \mathrm{m}$ ). The droplets (viewed along the $E$-field direction through transparent ITO electrodes) were subjected to $E=180 \mathrm{~V} \mathrm{~mm}^{-1}$ for around $1 \mathrm{~min}$ and after that the $E$
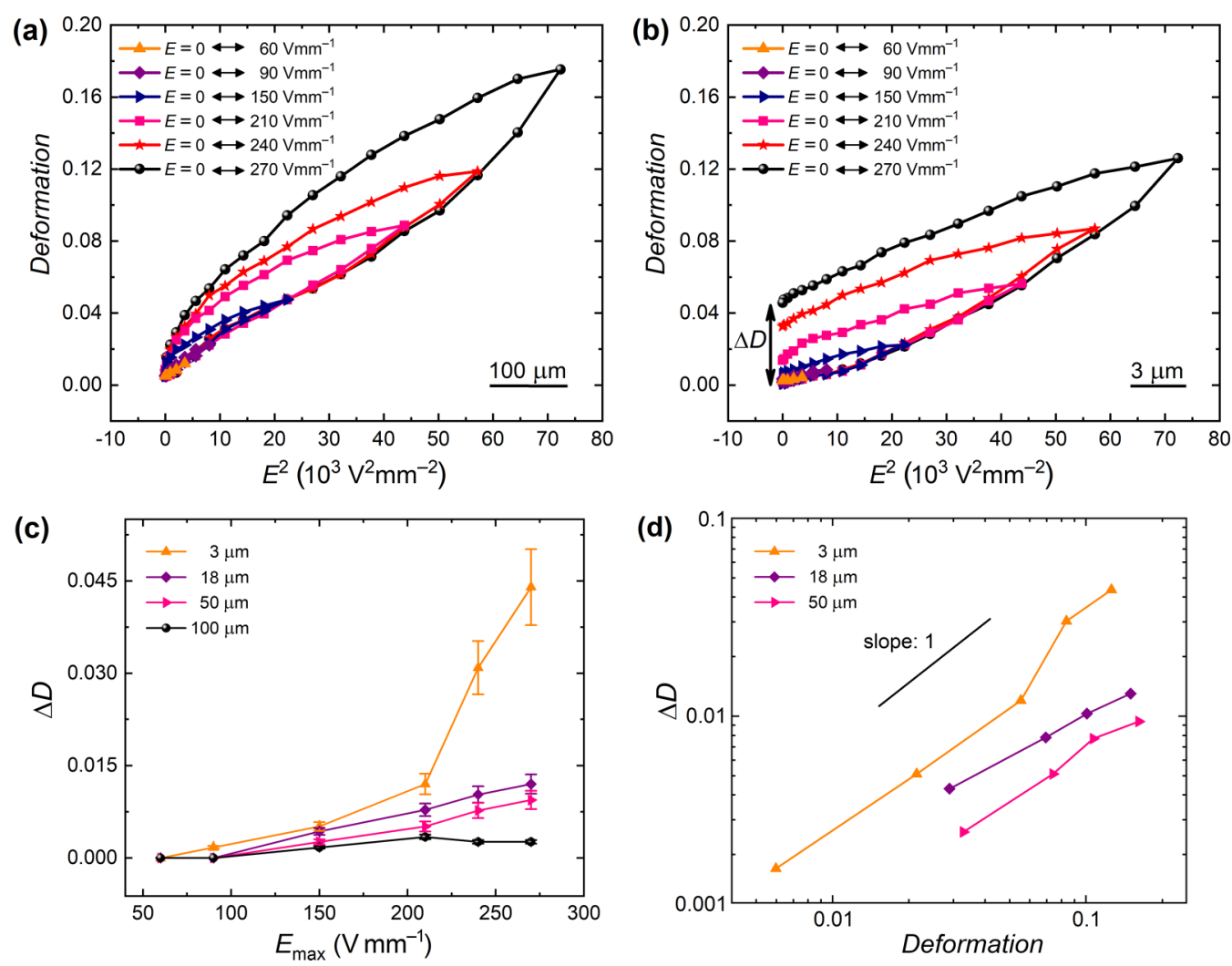

FIG. 7. Effect of the $E$ field on the hysteresis area of droplet deformation: Droplets of diameter $\sim 4$ mm fully covered by PE particles with different particle sizes (a) $\sim 100 \mu \mathrm{m}$ and (b) $\sim 3 \mu \mathrm{m}$ were subjected to different $E$-field strengths for six cycles. (c) Residual deformation ( $\Delta D$ ) of the four particle-covered droplets plotted against the maximum $E$-field strength. (d) A log-log plot of $\Delta D$ versus $D$ for the three droplets (covered with particles in the size range of 3-50 $\mu \mathrm{m}$ ) for which the residual deformation is observed. 
(a)

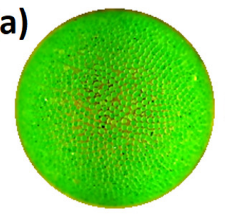

(b)

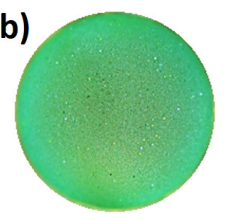

(c)

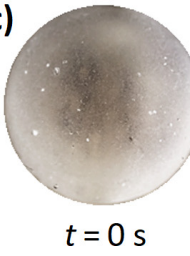

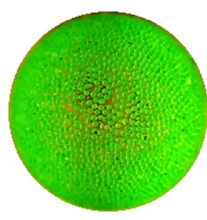
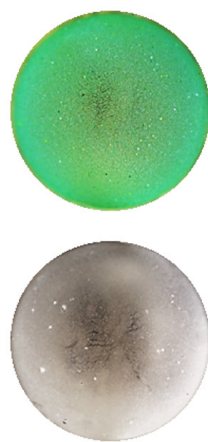

$t=3 \mathrm{~s}$
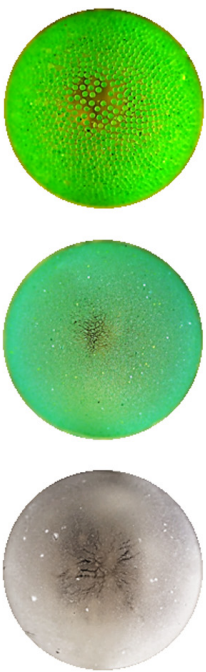

$t=8 \mathrm{~s}$
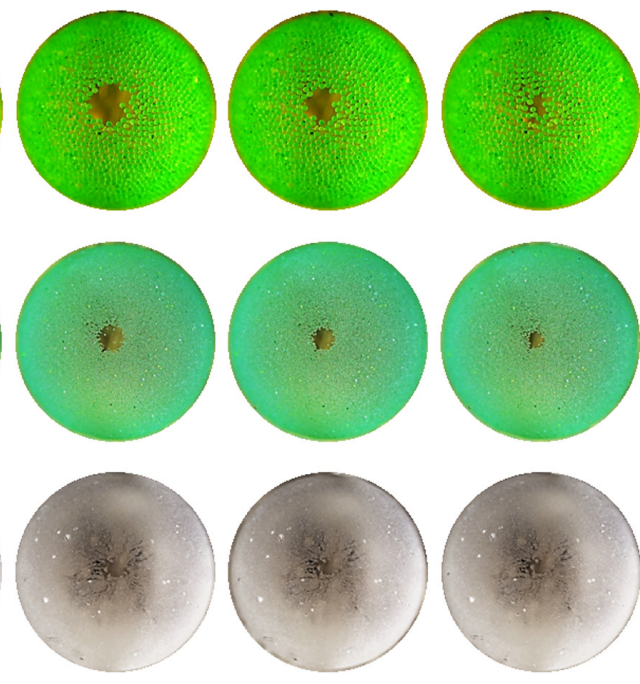

$t=73 \mathrm{~s}$

$t=93 \mathrm{~s}$

$t=276 \mathrm{~s}$

FIG. 8. Opening and closing of PE particle shells made of (a) $100 \mu \mathrm{m}$, (b) $18 \mu \mathrm{m}$, and (c) $3 \mu \mathrm{m}$. Initially at $t=0 \mathrm{~s}$, the particle shells were spherical in shape. Application of the $E$ field of strength $180 \mathrm{~V} \mathrm{~mm}^{-1}$ (up to $t=73 \mathrm{~s}$ ) resulted in (a) and (b) liquifying and (c) fracturing of a particle shell leading to formation of a hole at the droplet's electric pole. After the $E$ field was turned off, the droplet relaxed back and opening area at pole decreased by around $75 \%, 50 \%$, and $25 \%$ for the shells made of 100-, 18-, and 3- $\mu \mathrm{m}$ PE particles, respectively.

field was turned off enabling droplets to relax. As shown in Figs. 8(a) and 8(b), the application of the $E$ field to the droplets covered with 100- and 18- $\mu \mathrm{m}$ PE particles resulted in formation of a small opening at the electric pole of each droplet. The particles separated very easily from one another, and the particle layer liquified. The openings had circular shapes without any fracturing features. After switching off the $E$ field, the droplets relaxed returning nearly to the spherical shapes. The size of the opening was reduced by around $75 \%$ and $50 \%$ for the shells made of 100 - and $18-\mu \mathrm{m}$ PE particles, respectively. The behavior of the shell composed of the smallest PE particles was different. Unlike the two other shells, the shell made of 3- $\mu \mathrm{m}$ PE particles fractured during the droplet compressive deformation [see Fig. 8(c)]. A small hole was formed in the particle shell that recovered very little (around 25\%) after turning off the $E$ field, leaving unhealed cracks. The reason for this could be the increased cohesive force between the particles (which is inversely proportional to particle
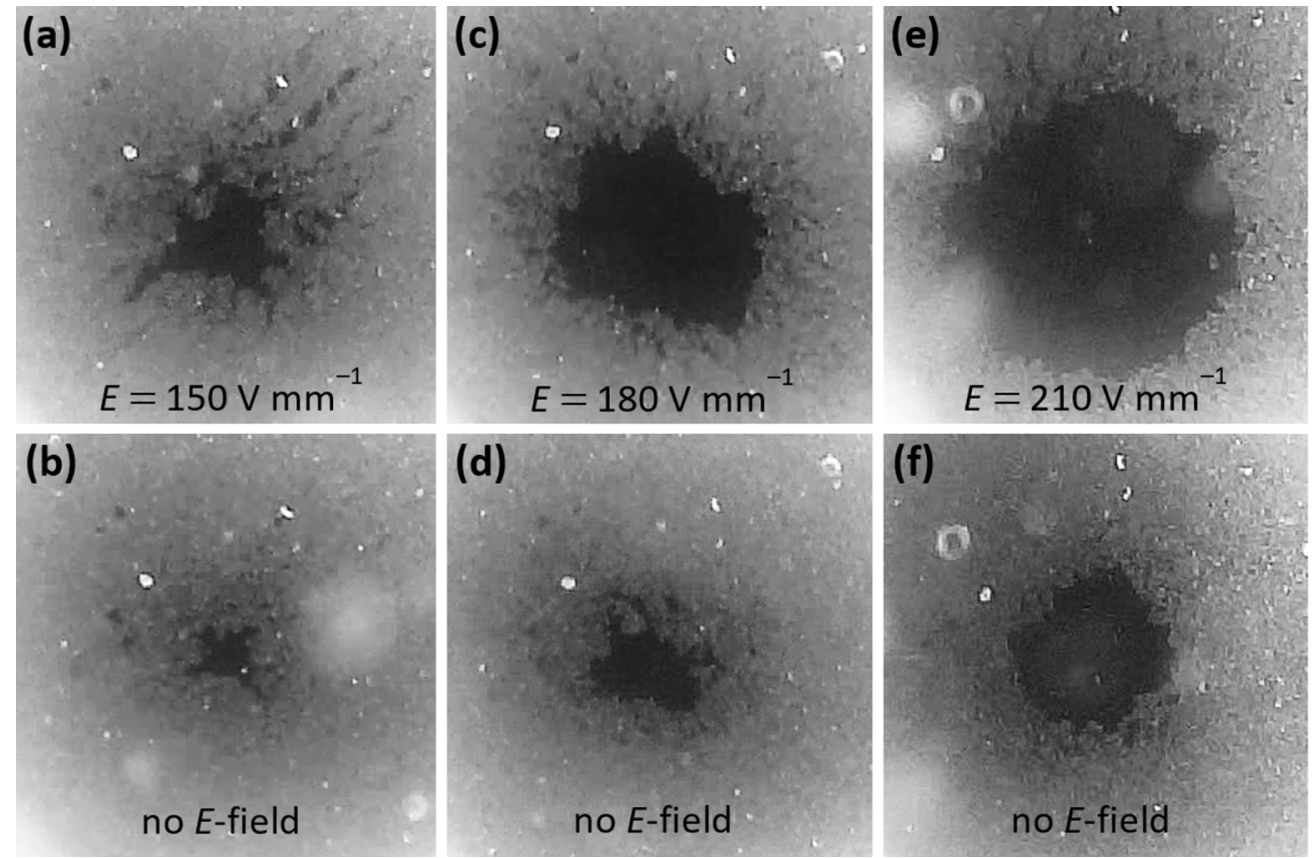

FIG. 9. Opening and closing of clay mineral particle shell under different $E$-field strengths. The camera view is along the direction of the applied $E$ field. The droplet is subjected to an $E$-field strength of (a) $150 \mathrm{~V} \mathrm{~mm}^{-1}$, (c) $180 \mathrm{~V} \mathrm{~mm}^{-1}$, (e) $210 \mathrm{~V} \mathrm{~mm}^{-1}$, and in panels (b), (d), and (f) the $E$ field is turned off, respectively. See also the corresponding Movie S1 in the Supplemental Material [52]. 
size [59]) that prevents the individual particle's rearrangement during the droplet relaxation and/or the increase in packing density of particles forming the shell. We know from our previous experiments that clay mineral particles suspended in oils form cohesive films [52]. Therefore, we decided to perform an additional experiment in which we formed a clay mineral particles shell on a silicone oil droplet to compare its behavior with that of the PE particle shells.

In Fig. 9, we present the results of an experiment on a droplet covered with $\mathrm{Li}$ fluorohectorite clay mineral particles (average size $\sim 5 \mu \mathrm{m}$ ) subjected to different $E$-field strengths $\left(150-210 \mathrm{~V} \mathrm{~mm}^{-1}\right)$. Typically, clay mineral particles adhere to each other strongly when suspended in oil due to the presence of small amounts of water (in the clay). Therefore, they form an elastic membrane that fractures under the load unlike a sheet made of noncohesive particles that liquefies under the load [60]. When a weak $E$ field $\left(150 \mathrm{~V} \mathrm{~mm}^{-1}\right)$ was applied, the clay particle shell fractured as shown in Fig. 9(a). The fracturing is similar to that observed for the shell made of the smallest (3- $\mu \mathrm{m})$ PE particles presented in Fig. 8(c). At stronger $E$ fields $\left(180-210 \mathrm{~V} \mathrm{~mm}^{-1}\right.$ ), the EHD flows ripped off small fragments of clay particle clusters and redistributed them within the droplet's surface and a nearly spherical opening was formed on the droplet's electric pole with a size depending on the strength of the $E$ field (see Figs. 9(c) and 9(e) and Supplemental Material [52] Movie S1). After turning off the $E$ field the particle-covered droplet relaxed. However, the droplet did not return to the initial spherical shape. Similar to the experiments with small PE particles [Figs. 7(b) and 8(c)], the clay mineral particles jammed and formed an arrested shell, leaving the hole in the particle shell. The size of the hole depended on the magnitude of the $E$ field, i.e., the stronger the applied $E$ fields, the larger the remaining hole in the shell after $E$ field removal. The high cohesiveness between particles inhibits particle relocation when the droplet with the particle shell relaxes after turning off the $E$ field. This, in turn, leads to particle jamming before the opening in the shell is healed.

\section{CONCLUSIONS}

We used $E$ fields to study the behavior of both a particlecovered droplet and a particle shell under compressive stress. Unlike the mechanical approaches (e.g., using a micropipette or a micromanipulator $[61,62])$, the $E$-field methods enable contactless application of stress and measurement of droplet deformation simultaneously with the examination of mechanical properties of the particle shell. We wondered how the particle coverage and the particle size as well as the strength of the $E$ field influenced the magnitude of the droplet deformation. The experimental results indicate that adding particles to a droplet interface drastically changes the magnitude of the droplet deformation. With the PIV experiments we have shown that the straining flows were suppressed by the addition of particles to the droplet's surface, which, in turn, enabled for generation of greater electric stress acting on the droplet. We also performed theoretical calculations to capture the upward bending trend of the magnitude of droplet deformation as a function of the particle coverage. Our experimental results on the particle coverage dependency of the magnitude of deformation differ from those presented by Ouriemi and
Vlahovska [22]. The researchers observed that the magnitude of the droplet deformation becomes less sensitive to the particle coverage at high coverages. This is not the case here, i.e., the magnitude of deformation clearly increases with the particle coverage until the droplet is entirely covered. Perhaps, the difference in the observations originates from the fact that Ouriemi and Vlahovska studied deformation of droplets unattached to any surface and the measurements were performed in the narrower range of $E$-field strengths. This would require further investigations.

We also found that the magnitude of the deformation is not retraceable during the electric-field sweeping, i.e., the strain-stress curves form a hysteresis loop with the area increasing with the increase in the $E$-field strength. We observed that during the droplet compression and recovery, the particles relocate separately or through particle gliding and rotate dissipating the energy. The particle rotation and gliding were documented as contributing to the friction by Mikkelsen et al. [27]. In addition, shells composed of small particles fractured during the droplet compression, indicating that they form cohesive films, which greatly affected droplet's deformation and relaxation.

The smallest particles were more prone to jamming and formed arrested shells on relaxed droplets. Stable nonspherical droplets and bubbles created by the interfacial jamming of arrested shells have been studied by other researchers $[12,29,63]$. However, in many studies the jammed state was achieved by increasing the particle concentration (by addition of particles to the interface or coalescing particle-covered droplets). Here, the jamming occurs due to the change in the particle arrangement, whereas the particle concentration remains unchanged. We believe, that the electric method demonstrated here can be used as a noncontact indenter to study and understand the stability of curved colloidal or granular crystals and amorphous particle shells under load [64].

The results of our studies are important for developing an understanding of the mechanics and rheology of monolayered colloidal and granular shells formed on droplets (or other curved interfaces) and the surface particle organization as well as the behavior of particle-covered droplets probed by induced stress. We demonstrated that $E$-field-induced droplet deformation was accompanied by structural and morphological changes in the particle shell. This can be exploited in multiple ways, for example, to form and study buckled armored droplets and plastic rearrangements of the particle shell as well as to investigate fracturing of cohesive films on curved surfaces.

The results of our research go beyond the academic sphere and have practical relevance. For example, a particle-covered droplet can be used as a miniaturized optical diaphragm with an adjustable aperture [46]. Controlling reliably the light passage through such a diaphragm requires the knowledge on mechanical properties of a particle shell that opens and closes $v i a$ application of an $E$ field. With the results presented here, we can now better design such a responsive optical element.

To further extend the research presented here, we suggest the future experimental studies to investigate the influence of particle properties (e.g., electrical conductivity and particle packing) on the stability and mechanics of particle shells under $E$ fields. 


\section{ACKNOWLEDGMENTS}

This research was funded by the Polish National Science Centre through PRELUDIUM (Grant No. 2019/35/ N/ST5/02821) and OPUS (Grant No. 2015/19/B/ST3/03055) and programmes. We also thank the Research Council of Norway for its support through the Centres of Excellence funding scheme, Project No. 262644.
K.K. initiated the project, designed, and performed all of the experiments. T.H. performed the theoretical calculations. K.K. authored the first draft of the paper. K.K., T.H., and Z.R. took part in discussions towards the finalization of the paper. All authors have read and agreed to the published version of the paper.

The authors declare no conflict of interest.
[1] E. Dickinson, Curr. Opin. Colloid Interface Sci. 15, 40 (2010).

[2] T. Bollhorst, K. Rezwan, and M. Maas, Chem. Soc. Rev. 46, 2091 (2017).

[3] D. Venkataramani, A. Tsulaia, and S. Amin, Adv. Colloid Interface Sci. 283, 102234 (2020).

[4] C. L. G. Harman, M. A. Patel, S. Guldin, and G.-L. Davies, Curr. Opin. Colloid Interface Sci. 39, 173 (2019).

[5] J. Lee and T. Babadagli, J. Dispers. Sci. Technol. 41, 2048 (2020).

[6] A. Mikkelsen, K. Khobaib, F. K. Eriksen, K. J. Måløy, and Z. Rozynek, Soft Matter 14, 5442 (2018).

[7] P. Dommersnes, Z. Rozynek, A. Mikkelsen, R. Castberg, K. Kjerstad, K. Hersvik, and J. O. Fossum, Nat. Commun. 4, 2066 (2013).

[8] V. O. Ikem, A. Menner, T. S. Horozov, and A. Bismarck, Adv. Mater. 22, 3588 (2010).

[9] S. N. Yin, C. F. Wang, S. S. Liu, and S. Chen, J. Mater. Chem. C 1, 4685 (2013).

[10] J. Wang et al., Lab Chip 17, 1970 (2017).

[11] R. Bielas, D. Surdeko, K. Kaczmarek, and A. Józefczak, Colloids Surf., B 192, 111070 (2020).

[12] Z. Rozynek, A. Mikkelsen, P. Dommersnes, and J. O. Fossum, Nat. Commun. 5, 3945 (2014).

[13] Z. Rozynek and A. Józefczak, Eur. Phys. J. ST 225, 741 (2016).

[14] V. N. Manoharan, Science 349, 1253751 (2015).

[15] W. T. Irvine, V. Vitelli, and P. M. Chaikin, Nature (London) 468, 947 (2010).

[16] Z. Rozynek, J. Banaszak, A. Mikkelsen, K. Khobaib, and A. Magdziarz, Soft Matter 17, 4413 (2021).

[17] F. Sicard and A. Striolo, Nanoscale 9, 8567 (2017).

[18] Z. Rozynek, R. Bielas, and A. Józefczak, Soft Matter 14, 5140 (2018).

[19] C. P. Whitby and E. J. Wanless, Materials 9, 626 (2016).

[20] K. Hwang, P. Singh, and N. Aubry, Electrophoresis 31, 850 (2010).

[21] J. K. Ferri, P. Carl, N. Gorevski, T. P. Russell, Q. Wang, A. Boker, and A. Fery, Soft Matter 4, 2259 (2008).

[22] M. Ouriemi and P. M. Vlahovska, Langmuir 31, 6298 (2015).

[23] C. Gu and L. Botto, Soft Matter 12, 705 (2016).

[24] R. B. Karyappa, S. D. Deshmukh, and R. M. Thaokar, Phys. Fluids 26, 122108 (2014).

[25] L. Becu and L. Benyahia, Langmuir 25, 6678 (2009).

[26] A. B. Pawar, M. Caggioni, R. Ergun, R. W. Hartel, and P. T. Spicer, Soft Matter 7, 7710 (2011).

[27] Y. Mei, G. Li, P. Moldenaers, and R. Cardinaels, Soft Matter 12, 9407 (2016).
[28] A. Mikkelsen, P. Dommersnes, and J. O. Fossum, Rev. Cub. Fis. 33, 47 (2016).

[29] M. Cui, T. Emrick, and T. P. Russell, Science 342, 460 (2013).

[30] Y. Rane, E. Foster, M. Moradiafrapoli, and J. O. Marston, Powder Technol. 338, 7 (2018).

[31] S. Y. Tan, R. F. Tabor, L. Ong, G. W. Stevens, and R. R. Dagastine, Soft Matter 8, 3112 (2012).

[32] D. Zang, J. Li, Z. Chen, Z. Zhai, X. Geng, and B. P. Binks, Langmuir 31, 11502 (2015).

[33] X. Liu et al., Science 365, 264 (2019).

[34] M. S. Abbasi, H. Farooq, H. Ali, A. H. Kazim, R. Nazir, A. Shabbir, S. Cho, R. Song, and J. Lee, Materials 13, 2984 (2020).

[35] A. Mikkelsen, P. Dommersnes, Z. Rozynek, A. GholamipourShirazi, M. d. S. Carvalho, and J. O. Fossum, Materials 10, 436 (2017).

[36] A. Mikkelsen, Z. Rozynek, K. Khobaib, P. Dommersnes, and J. O. Fossum, Colloid. Surf., A 532, 252 (2017).

[37] P. M. Vlahovska, Annu. Rev. Fluid Mech. 51, 305 (2019).

[38] Z. Rozynek, R. Castberg, A. Kalicka, P. Jankowski, and P. Garstecki, Arch. Mech. 67, 385 (2015).

[39] G. I. Taylor, Proc. R. Soc. 291, 159 (1966).

[40] D. Das and D. Saintillan, J. Fluid Mech. 810, 225 (2017).

[41] J. W. Ha and S. M. Yang, Phys. Fluids 12, 764 (2000).

[42] P. F. Salipante and P. M. Vlahovska, Phys. Fluids 22, 112110 (2010).

[43] D. Das and D. Saintillan, J. Fluid Mech. 914, A22 A22,(2021).

[44] M. Ouriemi and P. M. Vlahovska, J. Fluid Mech. 751, 106 (2014).

[45] P. Siahcheshm, F. Goharpey, and R. Foudazi, Rheol. Acta 57, 729 (2018).

[46] Z. Rozynek, K. Khobaib, and A. Mikkelsen, ACS Appl. Mater. Interfaces 11, 22840 (2019).

[47] Y. Xue, H. Wang, Y. Zhao, L. Dai, L. Feng, X. Wang, and T. Lin, Adv. Mater. 22, 4814 (2010).

[48] A. Mikkelsen and Z. Rozynek, ACS Appl. Mater. Interfaces 11, 29396 (2019).

[49] M. Kaganyuk and A. Mohraz, Soft Matter 16, 4431 (2020).

[50] C. Monteux, J. Kirkwood, H. Xu, E. Jung, and G. G. Fuller, Phys. Chem. Chem. Phys. 9, 6344 (2007).

[51] H. Xu, S. Melle, K. Golemanov, and G. Fuller, Langmuir 21, 10016 (2005).

[52] See Supplemental Material at http://link.aps.org/supplemental/ 10.1103/PhysRevE.103.062605 for [brief description].

[53] J. R. Melcher and G. I. Taylor, Annu. Rev. Fluid Mech. 1, 111 (1969).

[54] D. A. Saville, Annu. Rev. Fluid Mech. 29, 27 (1997).

[55] J. Q. Feng and P. Roy, Proc. R. London, Ser A 455, 2245 (1999). 
[56] D. Vella, P. Aussillous, and L. Mahadevan, Europhys. Lett. 68, 212 (2004).

[57] A. Morshed, P. Dutta, M. R. Hossan, and R. Dillon, Phys. Rev. Fluids 3, 103702 (2018).

[58] M. Pavlin and D. Miklavcic, Biophys. J. 85, 719 (2003).

[59] H. Shi et al., KONA Powder Part. J. 35, 226 (2018).

[60] Z. Rozynek, P. Dommersnes, A. Mikkelsen, L. Michels, and J. O. Fossum, Eur. Phys. J.: Spec. Top. 223, 1859 (2014).
[61] O. I. Vinogradova, O. V. Lebedeva, and B. S. Kim, Annu. Rev. Mater. Res. 36, 143 (2006).

[62] M. P. Neubauer, M. Poehlmann, and A. Fery, Adv. Colloid Interface Sci. 207, 65 (2014).

[63] A. B. Subramaniam, M. Abkarian, L. Mahadevan, and H. A. Stone, Nature (London) 438, 930 (2005).

[64] K. Khobaib, A. Mikkelsen, T. Vincent-Dispotal, and Z. Rozynek, Soft Matter 17, 5006 (2021). 